\title{
Alterações salivares, sintomas bucais e qualidade de vida relacionada à saúde bucal em pacientes com doenças neuromusculares
}

\author{
Alteraciones salivares, síntomas bucales y \\ calidad de vida relacionada con la salud bucal en \\ pacientes con enfermedades neuromusculares
}

\section{Salivary changes, oral symptoms, and oral health-related quality of life in patients with neuromuscular diseases}

Andressa Kelly Alves Ferreira, Esp ${ }^{1 *}$

Isaura Freitas Teixeira de Argôlo, Esp ${ }^{1}$

Maria Sueli Marques Soares, $\mathrm{PhD}^{1}$

Ângelo Brito Pereira de Melo, PhD1

Recebido: 12 de abril de 2019 - Aceptado: 2 de setembro de 2019

Doi: https://doi.org/10.12804/revistas.urosario.edu.co/revsalud/a.8765

Para citar este artigo: Alves Ferreira AK, Freitas Teixeira de Argôlo I, Marques Soares MS, Brito Pereira de Melo A. Alterações salivares, sintomas bucais e qualidade de vida relacionada à saúde bucal em pacientes com doenças neuromusculares. Rev Cienc Salud. 2020;18(1): 82-95. https://doi.org/10.12804/revistas.urosario.edu.co/revsalud/a.8765

\section{Resumo}

Introdução: o presente estudo teve como objetivo avaliar o fluxo salivar, sintomas bucais e a qualidade de vida relacionada à saúde bucal em um grupo de pacientes com doenças neuromusculares. Materiais e Métodos: estudo transversal realizado com 30 indivíduos com doenças neuromusculares atendidos no ambulatório de neuroreabilitação de um hospital universitário brasileiro entre outubro e dezembro de 2017. Foram realizados anamnese e determinação do Fluxo Salivar em Repouso e do pH salivar. Para avaliação da qualidade de vida relacionada à saúde bucal foi empregado o questionário Oral Health Impact Profile-14. Aplicou-se análise descritiva dos dados e teste qui-quadrado, adotando-se o nível de significância de $5 \%(p<0.05)$. Resultados: a Esclerose Lateral Amiotrófica foi a doença de base mais prevalente (50\%). A média de Fluxo Salivar em Repouso foi de $0.44 \pm 0.34 \mathrm{ml} / \mathrm{min}$, sendo verificado hiperfluxo em $43.3 \%$ dos pacientes e hipossalivação em $3.3 \%$. A média do pH salivar foi $6.47 \pm 0.57$. Os sintomas orais mais frequentes foram disfagia (56.7\%) e xerostomia (43.3\%). A média do Oral Health Impact Profile-14

1 Universidade Federal da Paraíba, João Pessoa, Paraíba, Brasil.

Autor correspondente: andressa_kelly@msn.com 
foi de $5.54 \pm 4.93$ e $90 \%$ dos pacientes apresentaram algum impacto das condições bucais na qualidade de vida. Os sintomas de disfagia, xerostomia, ardência bucal e sialorreia apresentaram associação estatisticamente significante $(p<0.05)$ com a qualidade de vida relacionada à saúde bucal. As dimensões do Oral Health Impact Profile-14 que apresentaram maiores percentuais foram a inabilidade psicológica (14.5\%) e a limitação funcional (13.5\%). Conclusão: a maioria dos pacientes apresentaram disfunção de glândulas salivares e a presença de sintomas bucais influenciaram negativamente a percepção de qualidade de vida dos indivíduos com doenças neuromusculares.

Palavras-chave: doenças neuromusculares; saúde bucal; saliva; qualidade de vida; Unidade Hospitalar de Odontologia.

\section{Resumen}

Introducción: el presente estudio tuvo como objetivo evaluar el flujo salivar, los síntomas bucales y la calidad de vida relacionada con la salud bucal en un grupo de pacientes con enfermedades neuromusculares. Materiales y métodos: estudio transversal realizado con 30 individuos con enfermedades neuromusculares atendidos de forma ambulatoria en neurorrehabilitación en un hospital universitario brasilero entre octubre y diciembre de 2017. Fueron realizados anamnesis y determinación del flujo salivar en reposo y del pH salivar. Para la evaluación de calidad de vida relacionada con la salud bucal, fue empleado el cuestionario Oral Health Impact Profile-14. Se realizó un análisis descriptivo de los datos y el test chi-cuadrado, adoptándose el nivel de significancia de $5 \%(p<0.05)$. Resultados: la esclerosis lateral amiotrófica fue la enfermedad de base más prevalente (50\%). El promedio del flujo salivar en reposo fue de $0.44 \pm 0.34 \mathrm{ml} / \mathrm{min}$, con un hiperflujo del $43.3 \%$ de los pacientes e hiposalivación del $3.3 \%$. El promedio del pH salivar fue $6.47 \pm 0.57$. Los síntomas orales más frecuentes fueron disfagia (56.7\%) y xerostomía (43.3\%). El promedio del Oral Health Impact Profile-14 fue de $5.54 \pm 4.93$ y el $90 \%$ de los pacientes presentó algún impacto de las condiciones bucales en su calidad de vida. Los síntomas de disfagia, xerostomía, ardor bucal y sialorrea presentaron una asociación estadísticamente significante $(p<0.05)$ con la calidad de vida relacionada con la salud bucal. Las dimensiones del Oral Health Impact Profile-14 que presentaron mayores porcentajes fueron la inhabilidad psicológica (14.5\%) y la limitación funcional (13.5\%). Conclusión: la mayoría de los pacientes presentó una disfunción de las glándulas salivares, y la presencia de síntomas bucales influenció negativamente la percepción de la calidad de vida de los individuos con enfermedades neuromusculares.

Palabras clave: enfermedades neuromusculares; salud bucal; saliva; calidad de vida; unidad hospitalaria de odontología.

\section{Abstract}

Introduction: The aim of this study was to evaluate the salivary flow, the presence of oral symptoms, and the oral health-related quality of life perceptions among individuals with neuromuscular diseases. Materials and Methods: It is a cross-sectional study, with 30 individuals with neuromuscular diseases treated at a neurorehabilitation clinic of a Brazilian university hospital from October to December 2017. Anamnesis, assessment of resting salivary flow, and measurement of salivary $\mathrm{pH}$ were performed. The instrument Oral Health Impact Profile-14 was used to evaluate the oral health-related quality of life. Data were analyzed using descriptive analysis and chi-square test, using a $5 \%$ significance level ( $\mathrm{p}<0.05)$. Results: Amyotrophic Lateral Sclerosis was the most common disease in the group (50\%). The mean resting salivary flow was $0.44 \mathrm{ml} / \mathrm{min}$, where it was found $43.3 \%$ of high salivary flow and $3.3 \%$ of hypo-salivation. The mean salivary $\mathrm{pH}$ found was $6.47 \pm 0.57$, where $56.6 \%$ of the patients had neutral pH and $40 \%$ low $\mathrm{pH}$. The most prevalent oral symptoms were dysphagia (56.7\%) and xerostomia (43.3\%). 
The mean Oral Health Impact Profile-14 was $5.54 \pm 4.93$, with $90 \%$ of patients presenting some impact of oral conditions on quality of life. A statistically significant association $(p<0.05)$ between the quality of life and symptoms of dysphagia, xerostomia, oral burning, and sialorrhea was found. The Oral Health Impact Profile-14 dimensions with the highest percentages were psychological inability (14.5\%) and functional limitation (13.5\%). Conclusion: The majority of patients presented salivary gland dysfunction with high salivary flows. The presence of oral symptoms negatively influenced the individual's quality of life perceptions of individuals with neuromuscular diseases.

Keywords: Neuromuscular diseases; oral health; saliva; quality of life; Hospital Unit of Dentistry.

\section{Introdução}

$A_{\text {gem genética ou adquirida que apresentam dano primário ou secundário em nervos, }}^{\text {s doencas neuromusculares (DNM) compreendem um grande grupo de patologias de ori- }}$ músculos e/ou junções neuromusculares (1). Fazem parte desse grupo: a Atrofia Muscular Espinhal, as Polineuropatias Hereditárias ou Adquiridas, a ELA, a Miastenia Grave, as Distrofias musculares, entre outras. Podem se apresentar sob diferentes formas clínicas, possuindo em comum o caráter progressivo de perda de força e tônus muscular, acarretando impacto na qualidade de vida (Qv) dos indivíduos afetados (2).

Algumas DNM, devido ao seu padrão neurodegenerativo, podem ocasionar alterações no sistema estomatognático, como alterações de tônus e mobilidade da musculatura orofacial $(3,4)$. Além disso, os fármacos utilizados no tratamento das Dnm e suas comorbidades, a exemplo dos antidepressivos, anticonvulsivantes, imunossupressores e relaxantes musculares, podem repercutir nas estruturas orais, particularmente na função das glândulas salivares (5).

Alterações na qualidade e quantidade de saliva podem afetar a integridade dos tecidos orais, tendo como consequência maior predisposição à cárie dentária, doenças periodontais e candidose bucal. Também acarretam maior frequência de sintomas como ardor bucal, xerostomia, disgeusia e disfagia, e interferem na qv do indivíduo $(6,7)$.

O grau de desconforto e limitações que os agravos bucais podem ocasionar no bem-estar geral do indivíduo pode ser mensurado através de instrumentos avaliadores da Qualidade de Vida Relacionada à Saúde Bucal (QvRSB), sendo este um parâmetro cada vez mais importante nas investigações clínicas (8).

A saúde bucal é parte integrante e inseparável da saúde geral, devendo ser considerada um fator importante na recuperação e promoção de saúde de pacientes com doenças crônicas, considerando que a presença de condições clínicas subjetivas e objetivas relacionadas à saúde bucal podem prejudicar o prognóstico no paciente sistemicamente comprometido (9).

Nesse contexto, considerando a importância da saliva na homeostase bucal e a possibilidade de doenças neuromusculares interferirem no desempenho funcional das glândulas salivares, objetivou-se com o presente estudo avaliar a possível alteração na quantidade de 
saliva e a presença de sintomas bucais em pacientes com DNM, bem como avaliar a relação entre estas variáveis e a qualidade de vida destes pacientes.

\section{Materiais e métodos}

Coi realizado estudo observacional, descritivo e transversal com indivíduos portadores de DNM do Brasil, na cidade de João Pessoa/Paraíba, entre outubro e dezembro de 2017. A pesquisa foi aprovada pelo Comitê de Ética em Pesquisa do referido hospital (parecer de número 2.263.716), e todos os participantes assinaram o Termo de Consentimento Livre e Esclarecido formulado de acordo com a resolução 466/2012 do Conselho Nacional de Saúde/Ministério da Saúde.

O universo amostral do estudo foi de 69 pacientes e a amostra obtida por conveniência foi constituída de $43.3 \%$ deste total, sendo os pacientes selecionados aleatoriamente até completar $n=30$, considerando ser representativa do universo de pacientes com DNM, atendidos no serviço acima referido. Participaram do estudo indivíduos adultos (a partir dos 18 anos de idade), de ambos os sexos, com diagnóstico médico de doenças neuromusculares e que voluntariamente aceitaram participar da pesquisa. Foram excluídos indivíduos que não autorizaram sua inclusão, bem como aqueles que não possuíam condições físicas e/ou cognitivas para submeter-se ao estudo.

A coleta de dados foi executada por uma única examinadora devidamente calibrada. Inicialmente, foi realizada anamnese para registro de informações acerca da história médica dos pacientes, incluindo a identificação das doenças de base, presença de comorbidades, medicamentos utilizados e presença dos sintomas de xerostomia, ardência bucal, disfagia, alterações de paladar e sialorreia.

Para determinar a taxa de fluxo salivar em repouso (FSR) foi utilizado o método de pesagem, onde dois roletes de algodão previamente pesados em balança digital foram inseridos bilateralmente no assoalho bucal do paciente e retirados após 2 minutos para nova pesagem, sendo a diferença entre o peso inicial e final convertido em $\mathrm{ml} / \mathrm{min}$ (10). Estabeleceu-se sempre o mesmo horário para realização da sialometria (entre $8 \mathrm{~h}$ e $11 \mathrm{~h}$ ), sendo os participantes orientados a não escovar os dentes, comer ou beber, por pelo menos $1 \mathrm{~h}$ antes da coleta. Como valores de referência, considerou-se: hipossalivação ( $\leq 0.1 \mathrm{ml} / \mathrm{min})$, baixo fluxo $(0.11 \mathrm{a} 1.99 \mathrm{ml} /$ $\mathrm{min})$, fluxo normal $(0.2 \mathrm{a} 0.35 \mathrm{ml} / \mathrm{min})$ e hiperfluxo $(>0.35 \mathrm{ml} / \mathrm{min})(11,12)$.

Para determinação do $\mathrm{pH}$ salivar foi utilizado o método colorimétrico, onde uma fita medidora de $\mathrm{pH}$ (McolorpHast ${ }^{\mathrm{TM}}$, Alemanha) era colocada sobre a língua do paciente e a alteração de coloração da fita era comparada com escala colorimétrica padrão, sendo considerado parâmetro de normalidade $\mathrm{pH}=7$ (13). 
A presença e o grau de severidade da sialorreia, que se caracteriza pelo escape não intencional de saliva, foram avaliados através da Escala de severidade e frequência de sialorreia (14). Nesta, a severidade dos sintomas tem gradação de 1 a 4, e a frequência de 1 a 5, e o resultado da avaliação permite o estadiamento do paciente com os seguintes escores: sem sialorreia (I), sialorreia leve (II), moderada (III) ou severa (IV) (15).

Para verificação do impacto da saúde bucal na qualidade de vida dos indivíduos estudados utilizou-se o instrumento Oral Health Impact Profile - forma simplificada (oHIP-14), na versão traduzida e validada para o português brasileiro (16), onde é possível se obter uma estimativa da disfunção, desconforto e incapacidade relacionada à saúde bucal.O instrumento é composto por 14 questões, compreendendo sete dimensões: limitação funcional; dor física; desconforto psicológico; inabilidade física; inabilidade psicológica; inabilidade social e incapacidade. A análise das respostas foi realizada pelo método ponderado, onde valores ordinais foram codificados para cada item, sendo 0 = "nunca", 1 = "raramente", 2 = "às vezes", 3 = "quase sempre", 4 = "sempre". A pontuação de cada questão foi calculada multiplicando-se 0 valor da resposta pelo seu peso correspondente (17). Ao realizar a soma destes valores, foi obtida uma pontuação final para cada paciente, que variou de 0 a 28 pontos, gerando os seguintes escores: (онгр-14 = 0) sem impacto; $(0<$ oнIP-14 $\leq 9)$ baixo impacto; $(9<$ oнIP- $14 \leq 18)$ médio impacto e, $(18$ $<$ oHIP-14 $\leq 28$ ) alto impacto (18). As respostas do oнгp-14 também foram analisadas através do método de simples contagem, o qual, para cada paciente, foram contabilizadas as vezes em que apareceram os códigos 3 (quase sempre) e 4 (sempre), sendo este método utilizado para identificar os impactos funcionais e psicossociais mais graves (19).

Os dados obtidos foram processados e analisados estatisticamente no programa Statistical Package for Social Science (sPss), versão 21, sendo aplicadas análises descritivas e teste qui-quadrado, adotando-se o nível de significância de $5 \%(p<0.05)$.

\section{Resultados}

$\AA^{\text {s características gerais da amostra estudada (n=30) estão demonstradas na tabela } 1 .}$

Tabela 1. Características gerais da amostra estudada

\begin{tabular}{|c|c|c|}
\hline Caracterização da amostra & n (\%) & Média \pm desvio padrão \\
\hline \multicolumn{3}{|l|}{ Gênero } \\
\hline Masculino & $17(56.7))$ & \\
\hline Feminino & $13(43.3))$ & \\
\hline Idade & & $49.70 \pm 13.3$ \\
\hline
\end{tabular}




\begin{tabular}{lcc}
\hline \multicolumn{1}{c}{ Caracterização da amostra } & n (\%) & Média \pm desvio padrão \\
\hline Doenças neuromusculares & $15(50))$ \\
Esclerose Lateral Amiotrófica & $3(10))$ \\
Polineuropatia Desmielinizante Idiopática Crônica & $3(10))$ \\
Polineuropatia & $2(6.7))$ \\
Distrofia de Cinturas & $2(6.7))$ \\
Síndrome de Guillain-Barré & $2(6.7))$ \\
Paraparesia Espástica Hereditária & $2(6.7))$ \\
Amiotrofia Espinhal & $1(3.3))$ \\
Miopatia mitocondrial & $12(40))$ \\
\hline Comorbidades & $7(23.3))$ \\
Hipertensão & $3(10))$ \\
Diabetes & $2(6.7))$ \\
Hipotireoidismo & $1(3.3))$ \\
Epilepsia & $12(40))$ \\
\hline Fluxo salivar em repouso (ml/min.) & $7(23.3))$ \\
\hline Sintomas Bucais & \\
Disfagia & $13(23.3))$ \\
Disgeusia & $1(3.3))$ \\
Xerostomia & \\
\hline Ardência bucal & \\
\hline
\end{tabular}

Fonte: Dados diretos do estudo, João Pessoa, PB, 2017.

A média de idade entre os indivíduos da amostra estudada foi de $49.7 \pm 13.3$ anos e $56.7 \%$ (17) eram homens. Dentre as doenças de base apresentadas, a ela foi a mais prevalente, correspondendo a $50 \%$ (15) da amostra. Dos pacientes avaliados, $40 \%$ (12) possuíam pelo menos uma comorbidade, onde $23.3 \%$ (7) dos casos corresponderam à hipertensão arterial e $10 \%$ (3) a diabetes. Com relação ao uso de medicamentos constatou-se que mais de $40 \%$ (13) dos pacientes faziam uso de três a cinco medicamentos diferentes ao dia. Os medicamentos mais utilizados foram: agente antiglutamatérgico (43.3\%), antidepressivos (36.7\%) e anti-hipertensivos (26.6\%). 
Quanto ao fluxo salivar em repouso do grupo estudado, foi observada média de $0.44 \pm$ $0.34 \mathrm{ml} / \mathrm{min}$. Nota-se que $40 \%$ (12) dos pacientes apresentavam fluxo normal, $43.3 \%$ (13) tinham hiperfluxo, e em apenas $3.3 \%$ (1) havia a presença de hipossalivação (figura 1). 0 pH salivar teve média de $6.47 \pm 0.57,56.6 \%$ (17) dos pacientes apresentaram $\mathrm{pH}$ salivar neutro e $40 \%$ (12) pH baixo (pH 6.0).

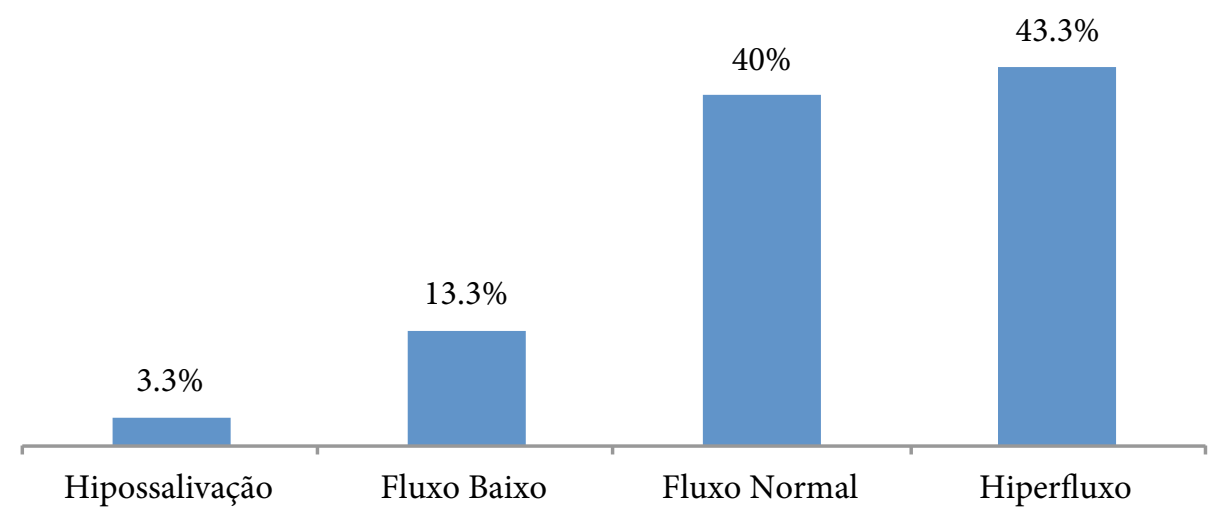

Figura 1. Distribuição de frequências dos pacientes com doenças neuromusculares quanto ao fluxo salivar

Fonte: Dados diretos do estudo, João Pessoa, PB, 2017.

Em relação à presença de sintomas bucais referidos pelos participantes, disfagia e xerostomia foram os mais prevalentes, sendo referidos por $56.7 \%$ (17) e $43.3 \%$ (13), respectivamente. Ardor bucal foi referido em apenas $3.3 \%$ (1) dos pacientes.

Quanto à presença e intensidade de sialorreia, a avaliação demonstrou que 26.7\% (8) do grupo apresentou algum grau de sialorreia, sendo o grau leve o mais observado (16.7\%).

No que se refere à QvRSB avaliada pelo oнIP-14, foi obtida uma média de $5.54 \pm 4.93$ através do método ponderado, correspondendo a um baixo impacto. Os resultados demonstraram que em $90 \%$ (27) dos pacientes houve algum impacto das condições bucais na qualidade de vida, sendo que a maioria (73.3\%) correspondeu a uma classificação de baixo impacto (figura 2).

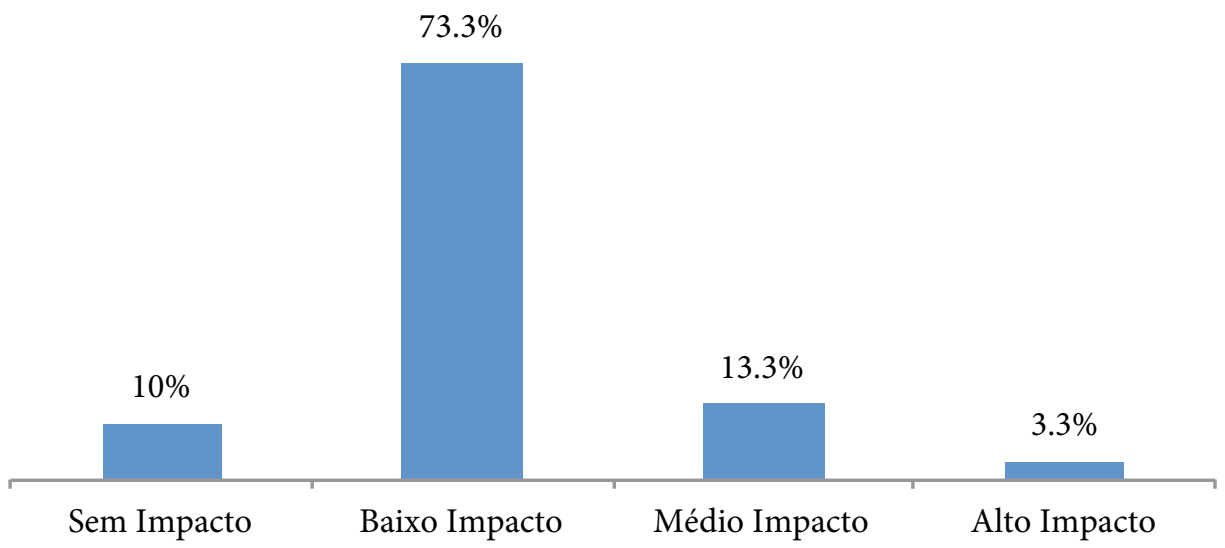

Figura 2. Impacto da saúde bucal na qualidade de vida avaliado pelo oHIP-14 
De acordo com os resultados do oHip-14 obtidos pelo método da simples contagem, foi observado que as dimensões que apresentaram maiores percentuais foram a inabilidade psicológica (14.5\%) e a limitação funcional (13.5\%), enquanto que desconforto psicológico foi a dimensão que menos influenciou (5\%) na QvRSB dos pacientes avaliados, conforme observado na figura 3.

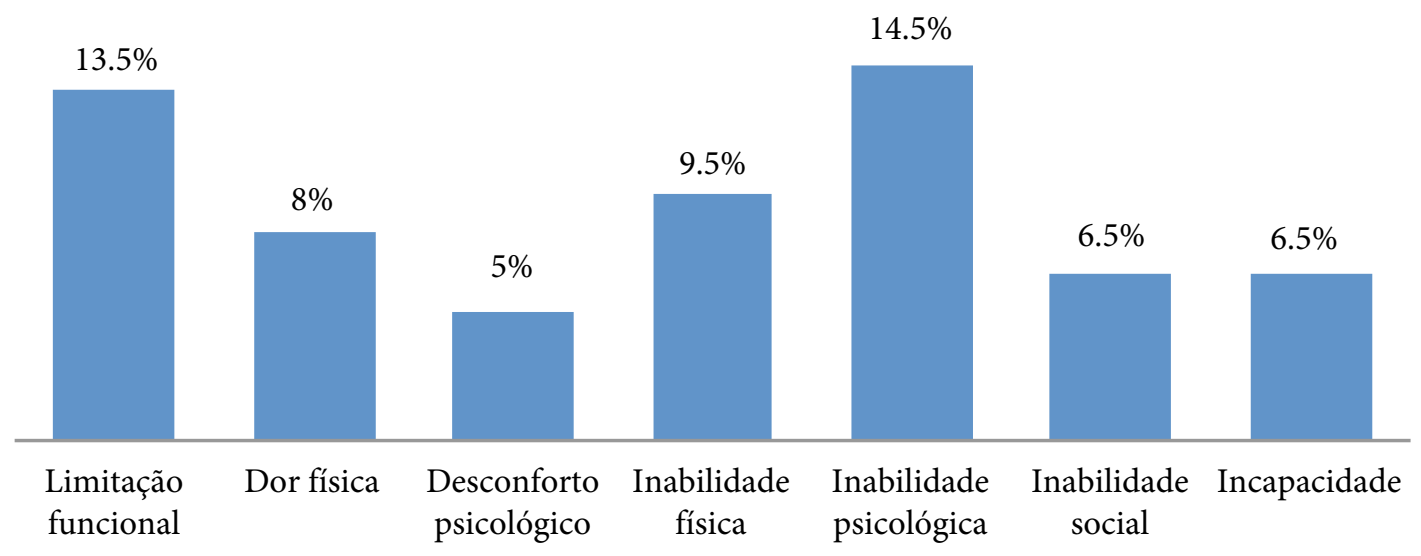

Figura 3. Análise do oHIP-14 por dimensões (método simples contagem)

Fonte: Dados diretos do estudo, João Pessoa, PB, 2017.

Na análise da gravidade dos impactos do oHIp-14 observou-se que a dimensão com maior média de frequências positivas, representadas pela resposta "sempre", foi a dimensão inabilidade psicológica (13\%). Em contrapartida, para os somatórios de frequências negativas relativas à resposta "nunca", os maiores percentuais foram para as dimensões incapacidade (88.5\%) e limitação funcional (86.5\%), de acordo com a tabela 2.

Tabela 2. Severidade do impacto por dimensões do oHIP-14 na amostra estudada

\begin{tabular}{lcc}
\hline & Severidade do impacto & \\
\hline & $\begin{array}{c}\text { Média \% / valores (n) } \\
\text { (respostas positivas “SEMPR”) }\end{array}$ & $\begin{array}{c}\text { Média \% / valores (n) } \\
\text { (respostas negativas “NUNCA") }\end{array}$ \\
Limitação funcional & $3.5(7-0)$ & $86.5(50-73)$ \\
Dor física & $6.5(3-10)$ & $43.5(37-50)$ \\
Desconforto psicológico & $5(7-3)$ & $53(43-63)$ \\
Inabilidade física & $6.5(13-0)$ & $61.5(50-73)$ \\
Inabilidade psicológica & $13(3-23)$ & $55(70-40)$ \\
Inabilidade social & $5(3-7)$ & $83(83-83)$ \\
Incapacidade & $6.5(10-3)$ & $88.5(80-97)$ \\
\hline
\end{tabular}

Fonte: Dados diretos do estudo, João Pessoa, PB, 2017. 
Foi observada associação estatisticamente significante entre disfagia e qualidade de vida ( $p=0.046$ ). Em $29.4 \%$ dos pacientes com disfagia houve médio e alto impacto na qualidade de vida. Na análise por dimensões do oHip-14, os sintomas de disfagia, xerostomia, ardência bucal e sialorreia foram as variáveis que demonstraram correlações significativas.

\section{Discussão}

$D^{\circ}$ e acordo com os resultados apresentados, a doença de base mais prevalente foi a ELA e houve uma média de idade de 49 anos. Este dado é similar ao observado por outros autores (20,21), que encontraram uma média de idade de aproximadamente 50 anos em pacientes acometidos por esta patologia.

Considerando a ocorrência de disfunção de glândulas salivares, foi observado elevado percentual de pacientes com hiperfluxo salivar e apenas $3.3 \%$ com hipossalivação. Este resultado corrobora com o resultado obtido em outro estudo (22), no qual a média do fluxo salivar foi ainda maior do que a observada na amostra estudada, demonstrando uma disfunção das glândulas salivares por excesso de estimulação ou hiperfunção. Uma possível justificativa para este achado se baseia no fato de que, no presente estudo, a ELA foi a doença de base predominante e que os pacientes com esta doença eram maioria entre aqueles que apresentaram aumento na taxa de fluxo salivar. Considerando que a etiopatogenia da ELA está fortemente associada a defeitos no transporte do glutamato, levando ao acúmulo deste neurotransmissor nos líquidos orgânicos (23), bem como considerando que a mucosa bucal e as glândulas salivares possuem receptores glutamatérgicos (24), pode-se supor que o acúmulo de glutamato também poderia estar presente na saliva do paciente com ELA e, assim, atuaria estimulando maior produção de fluxo salivar, particularmente nas glândulas salivares menores. Sugere-se que novos estudos sejam realizados para avaliar a concentração de glutamato na saliva de pacientes com ELA, visando contribuir para melhor entendimento de mecanismos que levam estes indivíduos a ter hiperfluxo salivar. Outra possibilidade para justificar o aumento na produção de saliva no grupo estudado, seria o comprometimento de vias regulatórias autonômicas, implicando em disfunção das glândulas salivares (25). No entanto, também tem sido observada redução na média do fluxo salivar de pacientes com DNM (26). A divergência dos resultados pode ser justificada considerando-se as diferenças metodológicas empregadas, bem como as características e condições dos indivíduos nos diferentes estudos.

No presente estudo, houve elevado percentual de pacientes com sialorreia. A literatura mostra que este é um achado comum em pacientes com patologias neurodegenerativas, como a ELA, uma vez que são observadas dificuldades no vedamento labial, com perda de saliva e 
alterações na dinâmica orofaríngea, ocasionando dificuldade na deglutição e consequente escoamento externo da saliva (27). Entretanto, a literatura aponta questionamentos quanto a presença de sialorreia em pacientes com distúrbios neuromusculares ser uma condição exclusivamente decorrente da presença de disfagia e de alterações neuromusculares, relacionadas à função dos músculos orofaciais inerentes à patologia, ou, se a disfunção das glândulas salivares com hiperfluxo salivar poderia ser um fator que contribuiria para a ocorrência de disfagia $(28,29)$. A constatação de hiperfluxo salivar observado entre os indivíduos da amostra, sugere que a condição pode contribuir para a ocorrência de escape salivar (sialorreia) nestes pacientes. Quanto ao grau de sialorreia, o encontrado no presente estudo foi grau leve, sendo provável que este dado tenha relação com o estágio inicial da doença, fase em que se encontravam os indivíduos estudados, não havendo um comprometimento intenso das estruturas orofaríngeas (3).

Dentre os sintomas bucais, a disfagia foi o mais prevalente e está em acordo com os achados de autores como Knuijt e colaboradores, em 2014 (30), sendo este um sintoma especialmente comum em pacientes com doenças neuromusculares com comprometimento bulbar, cuja característica já é bem conhecida na elA (31). Este é um fator importante para ser levado em consideração, pois a disfagia aumenta os riscos de o paciente contrair pneumonia por aspiração, especialmente quando a higiene bucal é negligenciada (32).

Outro sintoma referido pela maioria dos pacientes foi a xerostomia, que pode estar relacionada ao uso de medicamentos utilizados no tratamento de doenças neuromusculares e comorbidades. Dentre as várias drogas que podem causar xerostomia, destacam-se os anti-hipertensivos, diuréticos e os antidepressivos, que estão entre as classes de medicamentos mais utilizadas pelos participantes deste estudo. A prevalência da xerostomia também aumenta em relação ao número total de drogas que uma pessoa ingere, sendo estas medicações xerogênicas ou não (33), corroborando os achados desta pesquisa, onde a maioria dos pacientes utilizava de 3 a 5 medicamentos diferentes.

Não foi encontrada associação entre xerostomia e fluxo salivar, o que já é afirmado por autores como Hopcraft (2010), onde não há uma relação direta entre diminuição do fluxo e xerostomia, sendo portanto, variáveis independentes (34). Indivíduos que possuem fluxo salivar bastante diminuído podem não se queixar de boca seca, enquanto aqueles com salivação abundante também podem referir sensação de xerostomia, como visto nos pacientes do presente estudo, onde a xerostomia foi relatada por pacientes com fluxo salivar normal, diminuído e hiperfluxo. Um dos fatores apontados seria a qualidade salivar, considerando que alterações em sua composição química ou viscosidade podem desencadear a sensação de boca seca, mesmo na ausência de hipossalivação (31). Outra possibilidade seria a presença de alterações sensoriais na mucosa bucal, relacionadas ao comprometimento de mecanismos neuromusculares envolvidos nas DNM, sugerindo-se a realização de novos estudos para investigar essa possível relação. 
A análise do $\mathrm{pH}$ salivar demonstrou um percentual considerável de pacientes com $\mathrm{pH}$ ácido e este dado torna-se importante, uma vez que este é fator de risco para o surgimento de doenças bucais, como a cárie dentária (12). Um resultado interessante e inesperado foi que, embora a maioria dos pacientes tenham apresentado hiperfluxo salivar ou fluxo normal, houve redução do $\mathrm{pH}$ salivar. Poderíamos supor, que nestes pacientes, estaria havendo uma alteração qualitativa da saliva, a exemplo de uma maior concentração de glutamato nos casos de pacientes com ELA, influenciando na redução do pH salivar. Sugere-se que outros estudos sejam realizados para observar a influência do aumento de glutamato na saliva.

O questionário oнip-14 é bastante utilizado para indicar os aspectos da qualidade de vida mais afetados pelo estado de saúde bucal. Sendo um forte aliado no estabelecimento de melhores abordagens para atendimento integral ao paciente. Esse instrumento permite indicar as dimensões da Qv afetadas pela condição de saúde bucal, norteando os profissionais acerca das condições mais importantes para o paciente (16). A análise do oHIP-14 demonstrou que na amostra estudada houve impacto das condições de saúde bucal em quase todos os pacientes, embora este impacto tenha sido de baixo grau. Ressalta-se que as condições bucais dos pacientes estudados refletiram mais sobre a inabilidade psicológica, sendo um dado observado em todas as análises do oHIP-14, ou seja, as condições bucais interferem na condição psicológica.

$\mathrm{Na}$ análise por dimensões do oHIP-14, percebe-se que a xerostomia esteve associada a impactos na qualidade de vida nas dimensões de inabilidade física e desconforto/inabilidade psicológica. De acordo com alguns autores, a xerostomia afeta a qualidade de vida ao interferir no humor, na fala e na percepção gustativa $(35,36)$. Já os impactos na qualidade de vida relacionados à sialorreia foram referentes às dimensões: limitação funcional, inabilidade física e inabilidade social. Provavelmente esta relação se justifica pelas consequências desfavoráveis decorrentes da sialorreia, que seriam os distúrbios da fala, do sono e da mastigação, mau hálito, desidratação pela perda de fluidos e eletrólitos, e isolamento social (12). A disfagia demonstrou correlação com os domínios referentes à dor/inabilidade física e inabilidade psicológica, e esses impactos podem ser justificados especialmente pelo desconforto e diminuição do prazer ao se alimentar quando o indivíduo tem disfagia e, consequentemente, há declínio na qualidade de vida (28).

Considerando os resultados encontrados, percebe-se que a presença de hiperfluxo salivar foi frequente nos pacientes com DNM e houve impacto das condições de saúde bucal sobre a percepção de qualidade de vida, especialmente relacionado ao aspecto psicológico, como também os sintomas de xerostomia, disfagia e sialorreia foram os fatores mais relevantes nesta repercussão. Embora tenha sido um baixo impacto, ressalta-se a importância de investigar e monitorar condições bucais subjetivas e objetivas que possam repercutir negativamente na qualidade de vida de pacientes com doenças neuromusculares, contribuindo assim para a tomada de decisões clínicas dentro da equipe multiprofissional. 


\section{Contribuição dos autores}

\footnotetext{
Certifico que todos os autores contribuíram substancialmente com a concepção do artigo e que todos leram e aprovaram a versão final do mesmo.
}

\section{Conflito de interesses}

eclaramos não haver conflito de interesses.

\section{Referências}

1. Diniz GPC, Lasmar LMLBF, Gianetti JG. Doenças neuromusculares e instrumentos úteis na avaliação motora em crianças e adolescentes. Rev Med Minas Gerais. 2010;20(4):12-19.

2. Burns TM, Graham CD, Rose MR, Simmons Z. Quality of life and measures of quality of life in patients with neuromuscular disorders. Muscle Nerve. 2012;46(1):9-25.

3. Bergendal B, Mcallister A. Orofacial function and monitoring of oral care in amyotrophic lateral sclerosis. Acta Odontol Scand. 2017;75(3):1-10. Doi: 10.1080/00016357.2016.1276212

4. Mcgeachan AJ, Mcdermott CJ. Management of oral secretions in neurological disease. Pract Neurol. 2017;17(2):96-103. Doi: 10.1136/practneurol-2016-001515

5. Sivaramakrishnan G, Sridharan K. Adverse drug reactions in the oral cavity. Drugs Ther Perspect. 2016;32(7):297-303. Doi: 10.1007/s40267-016-0302-9

6. Ekström J, Castagnola M, Messana M. Saliva and the Control of Its Secretion. In: Ekberg O, editor. Dysphagia. Berlin: Springer; 2012. Doi: 10.1007/174_2011_481

7. Wayama MT, Aranega AM, Bassi APF, Ponzoni D, Garcia Junior IR. Grau de conhecimento dos cirurgiões-dentistas sobre Odontologia Hospitalar. Rev Bras Odont. 2014;71(1):4852. Doi: http://dx.doi.org/10.18363/rbo.v71i1.491

8. Bennadi D, Reddy CVK. Oral health related quality of life. J Int Soc Prev Community Dent. 2013;3(1):1-6. Doi: 10.4103/2231-0762.115700

9. Locker D, Matear D, Stephens M, Jokovic A. Oral health-related quality of life of a population of medically compromised elderly people. Community Dent Health. 2002;19(2):90-7.

10. Pupo DB, Bussoloti Filho I, Bianca ML, Gustavo PK. Proposta de um método prático de sialometria. Rev Bras Otorrinolaringol. 2002;68(2):219-222. Doi: 10.1590/S003472992002000200010

11. Fallahi A, Khadivi N, Roohpour N, Middleton AM, Kazemzadeh-Narbat M, Annabi N, et al. Characterization, mechanistic analysis and improving the properties of denture adhesives. Dent Mater. 2018;34(1):120-131. Doi: 10.1016/j.dental.2017.09.015 
12. Saleh J, Figueiredo ZMA, Cherubini K, Salum FG. Salivary hypofunction: An update on aetiology, diagnosis and therapeutics. Arch Oral Biol. 2015;60(2):242-255. Doi: 10.1016/j. archoralbio.2014.10.004

13. Silva JYB, Brancher JA, Duda JG, Losso EM. Mudanças do pH salivar em crianças após a ingestão de suco de frutas industrializado. RSBO. 2008;5(2):7-11.

14. Thomas-stonell N, Greenberg J. Three treatment approaches and clinical factors in the reduction of drooling. Dysphagia. 1988;3:73-78. Doi: 10.1007/bf02412423

15. Costa CC, Ferreira JB. Aplicação de toxina botulínica nas glândulas salivares maiores para o tratamento de sialorréia crônica. Rev Bras Cir Cabeça Pescoço. 2008;37(1):28-31.

16. Oliveira BH, Nadanovsky P. Psychometric properties of the Brazilian version of the Oral Health Impact Profile - short form. Community Dent Oral Epidemiol. 2005;33(4):307-314. Doi: $10.1111 / \mathrm{j} .1600-0528.2005 .00225 . \mathrm{x}$

17. Slade GD, Spencer, AJ. Development and evaluation of the Oral Health Impact Profile. Community Den Health. 1994;11(1):3-11.

18. Bastos RS, Carvalho ES, Xavier A, Caldana ML, Bastos JR, Lauris JR. Dental caries related to quality of life in two Brazilian adolescent groups: A cross-sectional randomised study. Int Dent J. 2012;62:137-143. Doi: 10.1111/j.1875-595X.2011.00105.x

19. Allen PF, Locker D. Do item weights matter? An assessment using the oral health impact profile. Community Dent Health. 1997;14:133-138.

20. FGA SP, Lima JMB, Alvarenga RP. Epidemiologia da Esclerose Lateral Amiotrófica Europa/América do Norte/América do Sul/Ásia. Discrepâncias e similaridades. Revisão sistemática da literatura. Rev Bras Neurol. 2009;45(2):5-10.

21. Bucheli M, Andino A, Montalvo M, Cruz J, Atassi N, Berry J, et al. Amyotrophic lateral sclerosis: Analysis of ALS cases in a predominantly admixed population of Ecuador. Amyotroph Lateral Sclerosis Frontotemporal Degener. 2014;15(1-2):106-13. Doi: $10.3109 / 21678421.2013 .852590$

22. Nakayama R, Nishiyama A, Matsuda C, Nakayama Y, Hakuta C, Shimada M. Oral health status of hospitalized amyotrophic lateral sclerosis patients: A single-centre observational study. Acta Odontol Scand. 2018;76(4):294-298. Doi: 10.1080/00016357.2017.1420228

23. Barber SC, Shaw JS. Oxidative stress in ALS: Key role in motor neuron injury and therapeutic target. Free Radic Biol Med. 2010;48(5):629-41. Doi: 10.1016/j.freeradbiomed.2009.11.018

24. Nijima A. Reflex effects of oral, gastrointestinal and hepatoportal glutamate sensors on vagal nerve activity. J Nutr. 2000;130(4):971S-3S. Doi: 10.1093/jn/130.4.971S

25. Araujo APQC, Araujo IP, Araujo AQC. Autonomic nervous system dysfunction in motor neuron diseases. J Rare Dis Res Treat. 2018;3(1):1-5.

26. Charchaflie RJ, Bustos Fernandez L, Perec CJ, Gonzalez E, Marzi A. Functional studies of the parotid and pancreas glands in amyotrophic lateral sclerosis. J Neurol Neurosurg Psychiatry. 1974;37:863-867.

27. Viana SMPO, Alvarenga P. Manifestações orofaríngeas na Esclerose Lateral Amiotrófica. Rev Neurocien. 2015;23(2):173-181. Doi: 10.4181/RNC.2015.23.02.928.9p 
28. Pellegrini A, Lunetta C, Ferrarese C, Tremolizzo L. Sialorrhoea: how to manage a frequente complication of motor neuron disease. EMJ Neurol. 2015;3(1):107-113.

29. Dand P, Sakel M. The management of drooling in motor neurone disease. Int J Palliat Nurs. 2010;16(11):560-4. Doi: 10.12968/ijpn.2010.16.11.80024

30. Knuijt S, Kalf JG, de Swart BJ, Drost G, Hendricks HT, Geurts AC, et al. Dysarthria and dysphagia highly prevalent among various types of neuromuscular diseases. Disabil Rehabil. 2014;36(15)1285-9. Doi: 10.3109/09638288.2013.845255

31. Paris G, Martinaud O, Petit A, Cuvelier A, Hannequin D, Roppeneck P, et al. Oropharyngeal dysphagia in amyotrophic lateral sclerosis alters quality of life. J Oral Rehabil. 2013;40:199204. Doi: 10.12968/ijpn.2010.16.11.80024

32. Müller F. Oral hygiene reduces the mortality from aspiration pneumonia in frail elders. J Dent Res. 2015;94(3):14-16. Doi: 10.1177/0022034514552494

33. Wolff A, et al. A Guide to Medications Inducing Salivary Gland Dysfunction, Xerostomia, and Subjective Sialorrhea. A systematic Review Sponsored by The World Workshop on Oral Medicine VI. Drugs R D. 2017;17:1-17. Doi: 10.1007/s40268-016-0153-9

34. Hopcraft MS, Tan C. Xerostomia: An update for clinicians. Aust Dent J. 2010;55:238-244.

35. Molania T, Alimohammadi M, Akha O, Mousavi J, Razvini R, Salehi R. The effect of xerostomia and hyposalivation on the quality of life of patients with type II diabetes mellitus. Electron Physician 2017;9(11): 5814-5819. Doi: 10.19082/5814

36. Niklander S, Veas L, Barrera C, Fuentes F, Chiappini G, Marshall M. Risk factors, hyposalivation and impact of xerostomia on oral health-related quality of life. Braz. Oral Res. 2017;31:1-9. Doi: 10.1590/1807-3107BOR-2017.vol31.0014 